\title{
Prevalence and risk factors for extended-spectrum $\beta$-lactamase- and AmpC-producing Escherichia coli in dairy farms
}

\author{
M. A. Gonggrijp, ${ }^{*}$ I. M. G. A. Santman-Berends, ${ }^{* 1}$ A. E. Heuvelink, ${ }^{*}$ G. J. Buter, ${ }^{*}$ G. van Schaik, ${ }^{*} \dagger$ J. J. Hage, ${ }^{*}$ \\ and T. J. G. M. Lam*† \\ ${ }^{*}$ GD Animal Health, PO Box 9, 7400 AA Deventer, the Netherlands \\ †Faculty of Veterinary Medicine, Utrecht University, 3508 TC Utrecht, the Netherlands
}

\section{ABSTRACT}

A cross-sectional study was conducted to evaluate the prevalence of extended-spectrum $\beta$-lactamase (ESBL)- and plasmid-mediated AmpC-producing Escherichia coli and associated risk factors in dairy herds. One hundred dairy herds were randomly selected and sampled to study the presence of ESBL- and AmpCproducing $E$. coli in slurry samples. The sensitivity of testing slurry samples for ESBL/AmpC herd status is less than $100 \%$, especially for detecting herds with a low ESBL/AmpC prevalence. Therefore, whereas herds that tested positive for ESBL/AmpC-producing E. coli in slurry were defined as positive herds, herds with negative slurry samples were defined as unsuspected. Isolates of ESBL- and AmpC-producing E. coli were further characterized by detection and typing of their ESBL/AmpC gene. At the initial sampling, a comprehensive questionnaire was conducted at the participating farms. The farmers were asked questions about management practices potentially associated with the ESBL/AMPC herd status. Also, data on antimicrobial purchases during 2011 were acquired to evaluate whether the animal-defined daily dose of antimicrobials per year at farm level was associated with the ESBL/ AmpC herd status. Multivariable logistic regression models were used to determine the association between management practices and the ESBL/AmpC herd status. Six months after the initial slurry sampling, 10 positive herds and 10 herds that had an unsuspected ESBL/AmpC herd status during the first visit were resampled. At each farm, slurry samples and feces from 24 individual cows were collected to evaluate within herd dynamics. During the first sampling, ESBL/ AmpC-producing E. coli were isolated from the slurry

Received March 4, 2016.

Accepted August 2, 2016.

${ }^{1}$ Corresponding author: i.santman@gdanimalhealth.com samples collected at $41 \%$ of the herds. In total, 37 isolates were further characterized, revealing 7 different ESBL genes ( $b l a_{\text {CTX-M-1,-2,-14,-15,-32,-55 }}$ and $\left.b l a_{\text {TEM-52 }}\right), 1$ plasmid-encoded AmpC gene ( $\left.b l a_{\mathrm{CMY}-2}\right)$, and 1 chromosomally encoded $a m p \mathrm{C}$ gene ( $a m p \mathrm{C}$ type 3$)$. The total animal-defined daily dose of antimicrobials per year at farm level was not significantly different between ESBL/AmpC-positive and unsuspected dairy herds. The use of third- and fourth-generation cephalosporins, however, was found to be associated with ESBL/AmpC status, with higher use of these antimicrobials resulting in a significant higher odds to be ESBL/AmpC-positive. Management factors that were associated with a higher odds of being ESBL/AmpC-positive were treatment of all cases of clinical mastitis with antimicrobials, a higher proportion of calves treated with antimicrobials, not applying teat sealants in all cows at dry off, and the use of a floor scraper. This last association, however, was considered a methodological effect rather than a true risk factor. On 5 of the 10 initially positive farms, no ESBL/AmpC-producing E. coli were cultured from the slurry or any of the individual cow samples collected during the second sampling. In 4 of the initially unsuspected farms, slurry or individual cow samples tested positive during the second sampling. In conclusion, ESBL/AmpC could frequently be cultured from slurry samples collected from Dutch dairy farms and the ESBL/AmpC genes carried by the isolates were consistent with those reported earlier. The use of thirdand fourth-generation cephalosporins appeared to be associated the ESBL/AmpC herd status.

Key words: dairy cattle, antimicrobial use, extendedspectrum beta-lactamase, antimicrobial resistance

\section{INTRODUCTION}

Enterobacteriaceae possessing genes for producing extended-spectrum $\beta$-lactamases (ESBL) or plasmid-mediated AmpC $\beta$-lactamases (AmpC) are an emerging problem and of great importance for public health. These bacteria show resistance to $\beta$-lactam 
antimicrobials, including third- and fourth-generation cephalosporins, which are categorized by the World Health Organization as critically important for human medicine (WHO, 2012). Enterobacteriaceae acquire ESBL by mutation or by plasmid-mediated horizontal gene transfer, which results in resistance to oxyiminocephalosporins. For example, the ESBL enzymes of the TEM and SHV families are mutant derivatives of established plasmid-mediated $\beta$-lactamases, the ESBL enzymes of the CTX-M family, however, are acquired from environmental bacteria (Carattoli et al., 2008; Hawkey and Jones, 2009).

The AmpC $\beta$-lactamases differ from ESBL in their ability to hydrolyze cephalosporins. Where ESBL are able to hydrolyze third- and fourth-generation cephalosporins, AmpC enzymes only hydrolyze cephalosporins of the third generation. Furthermore, AmpC $\beta$-lactamases are able to hydrolyze cephamycins, whereas ESBL cannot. In addition, a difference exists in the way these enzymes are inhibited: ESBL are inhibited by clavulanic acid, whereas AmpC enzymes are not susceptible to clavulanic acid (Ewers et al., 2012; Dierikx et al., 2013).

In the last decade, the spread of $\beta$-lactamases, and especially the enzymes of the CTX-M type, has rapidly emerged. Before the year 2000, most reports of infections with ESBL-producing Enterobacteriaceae concerned TEM and SHV and occurred in hospitals; currently CTX-M is the most dominant type found ( $\mathrm{Pi}-$ tout and Laupland, 2008; Hawkey and Jones, 2009; Scientific Advisory Group on Antimicrobials of the Committee for Medicinal Products, 2009). The ESBL are found in food-producing animals, companion animals, horses, and the environment (EFSA, 2011; Dierikx et al., 2012; Korzeniewska et al., 2013; Laube et al., 2014; von Salviati et al., 2015).

From previous research in a nonrepresentative group of dairy herds, there seems to be an association between the occurrence of ESBL-producing Escherichia coli and the use of third- and fourth-generation cephalosporins (Tragesser at al., 2006; Snow et al., 2012). From the study of Snow et al. (2012), several additional management factors influenced the risk of a herd being classified as ESBL/AmpC-positive. Studies on risk factors associated with the ESBL/AmpC herd status have, to our knowledge, not been conducted before in a large and randomly selected group of dairy herds. Therefore, the aim of our study was to evaluate the between and within herd prevalence of ESBL/AmpC-producing $E$. coli in dairy herds in the Netherlands. In addition, associations between the ESBL/AmpC herd status and (1) the antimicrobials purchased and (2) farm management was evaluated.

\section{MATERIALS AND METHODS}

\section{Definition of ESBL/AmpC Herd Status}

In this study, slurry samples on a herd level were evaluated instead of individual feces samples from all cattle, to classify herds with regard to the presence of ESBL/AmpC-producing E. coli. Slurry samples were defined as samples of fresh manure gathered from either the floor scraper or, if no scraper was present, from 5 different places on the slatted floor. Because, the slurry may not be sensitive enough to detect a small number of ESBL/AmpC-positive individual cows, herds with slurry samples that tested negative for ESBL/AmpCproducing $E$. coli were defined as unsuspected herds. Herds that tested positive for ESBL/AmpC-producing E. coli in the slurry sample were defined as positive herds.

\section{Herds and Sample Collection}

For the cross-sectional study that was conducted between September and December 2011, 500 herds were randomly selected from all dairy farms in the Netherlands with a conventional (nonorganic) farming system, using STATA 13.0 (StataCorp, 2014). Conventional and organic herds were distinguished based on information of the identification and registration system (RVO, Assen, the Netherlands) combined with information from the SKAL database (control organization for organic farming, Zwolle, the Netherlands). All 500 farmers were contacted by mail with the request to participate in our study and the first 100 farmers that responded and agreed were included. With this number of herds, the ESBL/AmpC herd prevalence could be determined, with an accepted absolute error of $10 \%$ and $95 \%$ confidence assuming a presence of ESBL/AmpC in 50\% of the herds (Win-episcope 2.0 Thrusfield et al., 2001).

During the first herd visit, one slurry sample was collected by using transport swabs with Amies medium (Beldico, Duiven, the Netherlands). When a floor scraper was present on the farm, the scraper was swabbed at different places. When no floor scraper was present, manure samples from 5 randomly selected places on the slatted floor were thoroughly mixed and sampled by using the transport swab. The samples were refrigerated transported to the laboratory where bacteriological culturing started within $24 \mathrm{~h}$ after collection of the samples.

Six months after the first sampling, 10 herds that tested positive and 10 herds that tested negative for ESBL/AmpC-producing E. coli during the first visit were randomly selected and visited a second time. 
During this second visit, a slurry sample was collected using the same method as during the first visit. Additionally, individual fecal samples from 24 randomly selected clinically healthy lactating cows were collected on each farm to obtain an estimate of the within-herd prevalence with an accepted error of $15 \%$ and $95 \%$ confidence (assuming a within-herd prevalence of $80 \%$ in positive herds). By selecting only lactating cows, the within-herd prevalence was applicable for dairy cows within this age category and production status.

The individual fecal samples were collected by rectal palpation using a new glove for each animal. Immediately after collection, the fecal samples was transferred into sterile containers. Both the slurry samples and the individual fecal samples were refrigerated at 2 to $8^{\circ} \mathrm{C}$ following collection and were immediately transported to the laboratory where bacteriological culturing started within $24 \mathrm{~h}$ after collection of the samples.

\section{Bacteriological Culture and Phenotypic ESBL/AmpC Testing}

At the laboratory, the samples were processed as described previously by Hordijk et al. (2013b) and EFSA (2011). Briefly, the slurry swabs were transferred into a tube with $10 \mathrm{~mL}$ of Luria-Bertani broth (Becton Dickinson and Company, Franklin Lakes, NJ) supplemented with $1 \mathrm{mg} / \mathrm{L}$ of cefotaxime (LBB+cef; SigmaAldrich, St. Louis, MO). The fecal samples from the individually sampled cows were processed similarly; a cotton swab was saturated in the fecal sample and then transferred to a tube with $10 \mathrm{~mL}$ of $\mathrm{LBB}+$ cef. Inoculated $\mathrm{LBB}+$ cef tubes were incubated aerobically overnight at $37^{\circ} \mathrm{C}$. Subsequently, all overnight cultures were streaked onto MacConkey agar (Becton Dickinson) supplemented with $1 \mathrm{mg} / \mathrm{L}$ of cefotaxime. After overnight incubation at $37^{\circ} \mathrm{C}$, all presumptive $E$. coli colonies were confirmed as E. coli using matrix-assisted laser desorption/ionization time-of-flight MS (Maldi Biotyper, Bruker Daltonics GmbH, Billerica, MA). Confirmed E. coli isolates (1 per sample, randomly selected) were examined for ESBL or AmpC production by combination disk diffusion tests using cefotaxime and ceftazidime with and without clavulanic acid (Becton Dickinson) according to CLSI (2011) guidelines. Additionally, a cefoxitin disk (30 $\mu \mathrm{g}$, Becton Dickinson) was included in the test to detect AmpC phenotypes. Isolates nonsusceptible to cefoxitin, according to CLSI criteria (zone diameter $\leq 17 \mathrm{~mm}$ ), were classified as AmpC-producers. Escherichia coli isolates producing ESBL/AmpC were stored at $-80^{\circ} \mathrm{C}$ in Microbank vials (Pro-lab Diagnostics, Austin, TX).

\section{Molecular Typing of ESBL/AmpC Genes}

Phenotypically confirmed ESBL- and AmpC-producing E. coli were further characterized to identify the $\beta$-lactamase genes. The aim of molecular typing of the ESBL/AmpC-positive isolates was to determine whether the types found had been encountered before and whether there was overlap with human types of ESBL/AmpC-producing E. coli. Isolation of DNA was performed with the DNeasy Blood \& Tissue kit (69506, Qiagen Benelux B.V., Venlo, the Netherlands). From each isolate 3 to 4 colonies, collected from a MacConkey agar plate with $1 \mathrm{mg} / \mathrm{L}$ of cefotaxime, were resuspended in $180 \mu \mathrm{L}$ of ATL buffer of the kit. The DNA isolation was performed according to the manual from the manufacturer.

With the use of the Check-MDR ct101 micro-array (10-0020, Check-Points B.V., Wageningen, the Netherlands), ESBL families ( $b l a_{\mathrm{CTX}-\mathrm{M}}, b l a_{\mathrm{TEM}}$ en $\left.b l a_{S H V}\right)$ and plasmid-mediated AmpC families ( $b l a_{\mathrm{CMY}}, b l a_{\mathrm{DHA}}$, $b l a_{\mathrm{FOX}}, b l a_{\mathrm{MOX}}, b l a_{\mathrm{ACC}}, b l a_{\mathrm{MIR}}$ en $\left.b l a_{\mathrm{ACT}}\right)$ were detected and identified. In addition, ESBL and non-ESBL $b l a_{\text {TEM }}$ and $b l a_{\mathrm{SHV}} \beta$-lactamases were differentiated and the identification of the different groups within $b a_{\text {TEM }}$ and $b a_{\mathrm{SHV}} \beta$-lactamases (A-L and $\mathrm{A}-\mathrm{E}$, respectively) were established.

Further determination of the ESBL/AmpC types was performed by PCR amplification followed by sequencing using different primers (Table 1). The PCR consisted of $5 \mathrm{~min}$ of denaturation at $95^{\circ} \mathrm{C}$, followed by 30 cycles of $30 \mathrm{~s}$ at $95^{\circ} \mathrm{C}, 30 \mathrm{~s}$ of annealing at temperatures (mentioned in Table 1), and $60 \mathrm{~s}$ of extension at $72^{\circ} \mathrm{C}$. Amplicons were purified and sequenced as described previously by Dierikx et al. (2010). All E. coli isolates with AmpC phenotypes that could not be attributed to the presence of a plasmid-mediated $\mathrm{AmpC}$ gene were tested for mutations in the AmpC promoter or attenuator region according to the same procedure. Sequences were compared with references in GenBank (www.ncbi. nlm.gov) and the Lahey database (Lahey, 2012).

\section{Calculation of the Animal-Defined Daily Dose per Year of Antimicrobials}

Veterinary service providers were asked to deliver all of the invoices from 2011 of the participating farmers. The invoices were digitalized by hand by one person and checked by a second person. From this information, the exact amount of antimicrobials and teat sealants that were supplied to each of the dairy herds during the study period was calculated. The antimicrobials were classified in 11 groups based on their mechanism of action, chemical structure, or spectrum of activity: ami- 
Table 1. Target genes, primer name, annealing temperature (Ta), primer sequence, and product size of the $8 \beta$-lactamase genes used for molecular $\beta$-lactamase or extended-spectrum $\beta$-lactamase typing of Escherichia coli isolates (Dierikx et al., 2012)

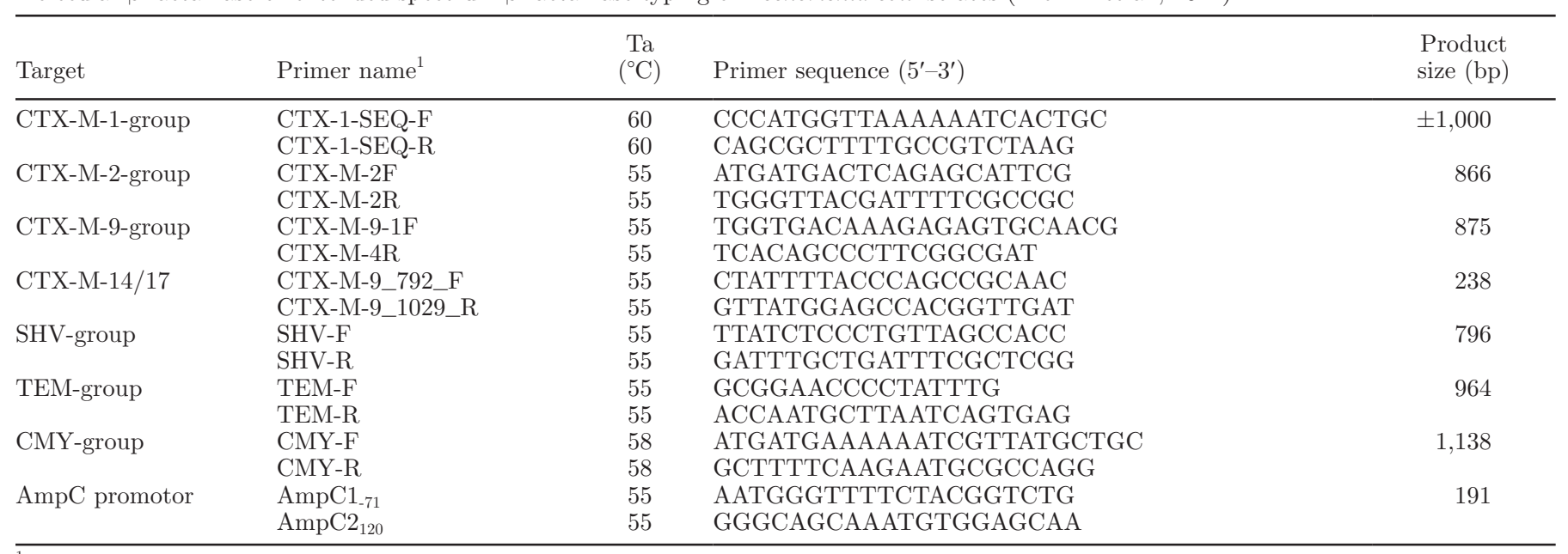

${ }^{1} \mathrm{~F}=$ forward, $\mathrm{R}=$ reverse.

noglycosides, amphenicols, first- and second-generation cephalosporins, third- and fourth-generation cephalosporins, combination preparations (benzylpenicillin/ neomycin/procaine benzylpenicillin, cephalexin/kanamycin and lincomycin/neomycin), fluoroquinolones, macrolides, penicillins (narrow and broad spectrum), polymyxins, tetracyclines, and trimethoprim/sulfamethoxazole.

The animal-defined daily dose per year calculated at farm level $\left(\mathbf{D D D A}_{\mathbf{F}}\right)$ was calculated following the standard operating procedures of The Netherlands Veterinary Medicines Authority (SDa, 2015). In short, to be able to calculate the DDDAF, additional data on herd size (provided by the Dutch Enterprise Agency, The Hague, the Netherlands), the standard weights of cattle in the Netherlands, and pharmacological information (the Faculty of Veterinary Medicine, Utrecht University) were acquired. For each herd, the amount of treatable weight $\left(\mathrm{DDA}_{\mathrm{kg}}\right)$ was calculated for the year 2011, by multiplying the amount of delivered product $\left(\right.$ product $\left._{i}\right)$ in 2011 with the registered amount of treatable weight per dose (milliliters, milligrams, grams, piece, pastille, and so on) of the delivered product ( $k g \_$treated $_{i}$; equation 1). Treatable weight was defined as the weight $(\mathrm{kg})$ of a cow that can be treated with 1 $\mathrm{U}$ of the product as described in the instructions of use developed by the manufacturer of the product:

$$
\mathrm{DDA}_{\mathrm{kg}}=\sum_{i=1}^{n} \text { product }_{i} \times k g_{-} \text {treated }_{i} .
$$

Subsequently, the DDDA $_{F}$ was calculated by dividing the amount of the total treatable weight by the total weight of cattle present per herd in $2011\left(k g_{\text {present }}\right)$, which was based on data of the identification and registration system combined with the standardized weights per age category (equation 2):

$$
\mathrm{DDDA}_{\mathrm{F}}=\frac{\mathrm{DDA}_{\mathrm{kg}}}{k g_{\text {present }}} .
$$

In these $\mathrm{DDDA}_{\mathrm{F}}$ calculations, it was assumed that all purchased antimicrobials were actually used to treat animals. Additionally, an average weight for each standardized age category was used.

Antimicrobial usage (AMU) had 3 different application methods, oral AMU (DDDAF,oral $)$, dry cow therapy AMU (DDDA $\left.A_{F, d r y}\right)$, and intramammary AMU $\left(\mathrm{DDDA}_{F, \text { mast }}\right)$, which were calculated seperately. These $\mathrm{DDDA}_{\mathrm{F}}$ were calculated in a similar way as the total $\mathrm{DDDA}_{\mathrm{F}}$, with the exception that for these calculations only the relevant groups of antimicrobials and age categories were included. In the $\mathrm{DDDA}_{\mathrm{F}, \mathrm{dry}}$, drying off 1 cow with 4 tubes was calculated as 4 . Additionally, the $\mathrm{DDDA}_{\mathrm{F}}$ was calculated for each of the 11 antimicrobial groups for each of the application methods.

\section{Questionnaire Data}

In the period between December 2011 and February 2012, all farmers were contacted by phone and a comprehensive questionnaire was conducted. The items included in the questionnaire are summarized in 8 categories of management practices in Table 2. The 2 interviewees were informed about the background of the study and were instructed to ask the questions in a similar way. The questionnaire is available on request. 
Table 2. Questionnaire topics potentially associated with extended-spectrum $\beta$-lactamase (ESBL)/AmpC in the study herds

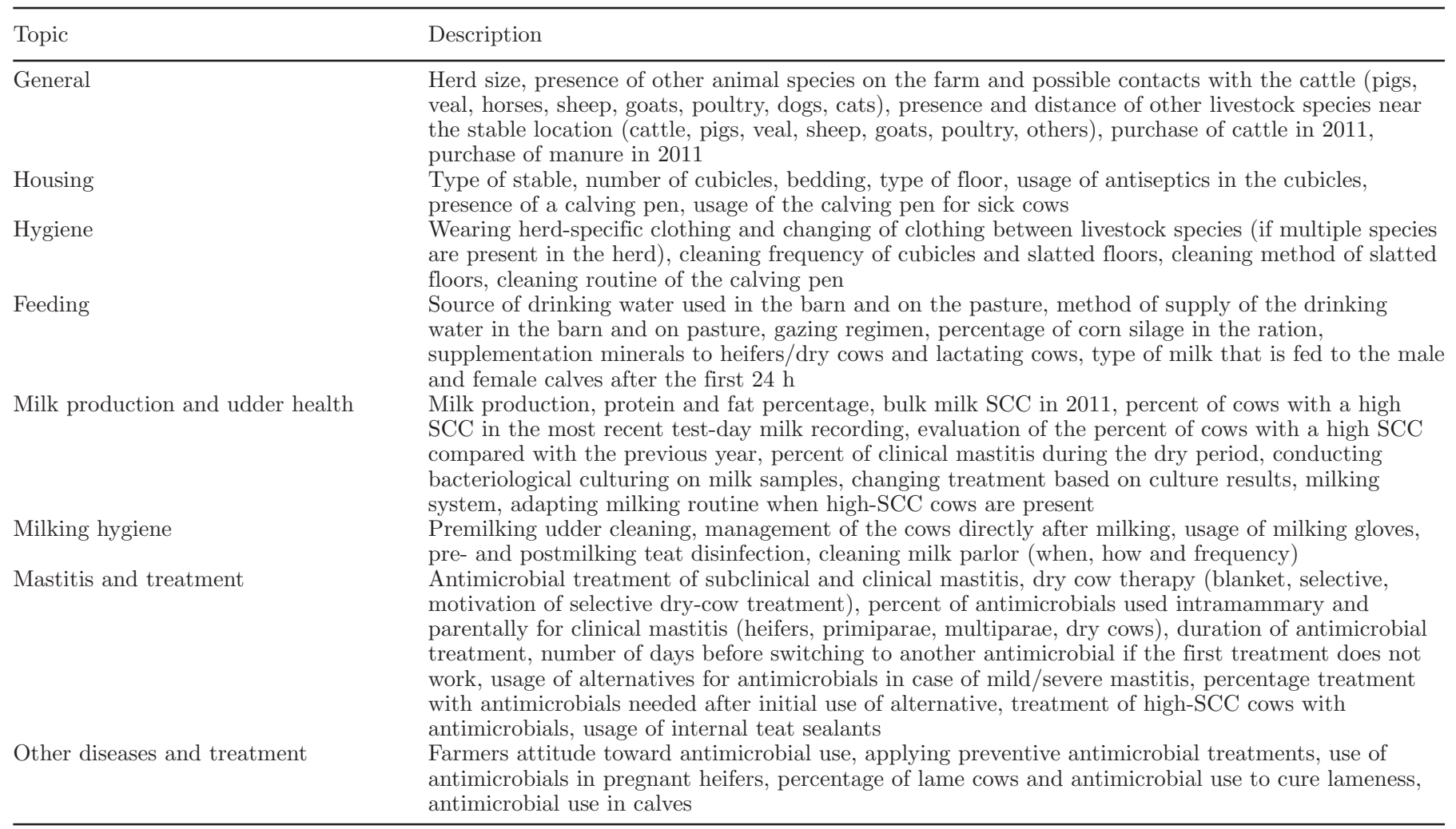

\section{Statistical Analysis}

All statistical analyses were carried out in Stata 13.0 (StataCorp, 2014). The herd prevalence during the initial sampling of ESBL/AmpC-producing E. coli in 100 dairy herds and the within-herd prevalence in 20 dairy herds at the second sampling were calculated using descriptive statistics.

To evaluate the association between the ESBL/ AmpC herd status and parameters such as AMU or other management factors, the ESBL/AmpC herd status during the first visit was used to classify the herds into 2 separate groups. The first sampling moment was chosen for classification because the data on AMU and management factors were collected at that point in time. A logistic regression model was used to evaluate the association between $\mathrm{DDDA}_{\mathrm{F}}$ (total $\mathrm{DDDA}_{\mathrm{F}}, \mathrm{DDDA}_{\mathrm{F} \text {,oral }}, \mathrm{DDDA}_{\mathrm{F}, \mathrm{dry}}$, and $\mathrm{DDDA}_{\mathrm{F}, \text { mast }}$ ) and the ESBL/AmpC herd status. Any $P$-values $\leq 0.05$ were considered significant. Likewise, the association between the ESBL/AmpC herd status, the use of different groups of antimicrobials (in 4 different $\mathrm{DDDA}_{\mathrm{F}}$ ), and the use of teat sealants was evaluated using the same model. In addition, to evaluate a possible associations between management factors and ESBL/AmpC herd status, a logistic regression model was used. All management factors that were potentially associated with the ESBL/AmpC herd status, were included and were first screened in a univariable analysis. Risk factors with an association with the ESBL/AmpC herd status at $P<0.25$ subsequently entered the multivariable model. With a forward selection and elimination procedure at each run, the variable with the lowest $P$-value in the univariable analysis entered the model until a final model was defined, in which all variables had $P \leq 0.05$, with the lowest Akaike information criterion value (Akaike, 1974). Confounding was monitored by the change $(>25 \%$ or 0.1 between values of -0.4 and 0.4 ) in the coefficient of a variable after removing another variable. Potential confounders re-entered in the model. The coefficient of determination (pseudo$\mathrm{R}^{2}$ ) was calculated to measure the proportion of variance explained by the model. To support the results of the questionnaire, the use of teat sealants, available from both the questionnaire and the invoices from the veterinary practice, were each evaluated in the logistic regression model.

\section{RESULTS}

\section{Prevalence of ESBL/AmpC-Producing E. coli Between and Within Herds}

The 100 dairy herds housed a median of 89 cows (>2 yr; interquartile range $=70-126)$ and had a me- 
Table 3. Isolation of extended-spectrum $\beta$-lactamase (ESBL)/AmpC-producing Escherichia coli from slurry samples and manure samples from 24 individual cows, collected on 20 dairy herds during 2 visits

\begin{tabular}{|c|c|c|c|}
\hline \multirow[b]{2}{*}{ Farm } & \multirow[b]{2}{*}{$\begin{array}{l}\text { First visit, slurry } \\
\text { test-result }^{1}\end{array}$} & \multicolumn{2}{|c|}{ Second visit } \\
\hline & & Slurry test-result & $\begin{array}{l}\text { Number of positive cows } \\
\text { (ESBL/AmpC) }\end{array}$ \\
\hline 1 & ESBL-positive & Negative & $3(2 / 1)$ \\
\hline 2 & ESBL-positive & Negative & 0 \\
\hline 3 & ESBL-positive & Negative & 0 \\
\hline 4 & ESBL-positive & Negative & 0 \\
\hline 5 & ESBL-positive & Negative & 0 \\
\hline 6 & ESBL-positive & Negative & 0 \\
\hline 7 & ESBL-positive & ESBL-positive & 0 \\
\hline 8 & ESBL-positive & AmpC-positive & $1(0 / 1)$ \\
\hline 9 & ESBL-positive & AmpC-positive & $24(0 / 24)$ \\
\hline 10 & ESBL-positive & ESBL-positive & $24(24 / 0)$ \\
\hline 11 & Negative & Negative & 0 \\
\hline 12 & Negative & Negative & $1(1 / 0)$ \\
\hline 13 & Negative & Negative & 0 \\
\hline 14 & Negative & Negative & $1(1 / 0)$ \\
\hline 15 & Negative & ESBL- and AmpC-positive & $4(4 / 0)$ \\
\hline 16 & Negative & Negative & 0 \\
\hline 17 & Negative & Negative & 0 \\
\hline 18 & Negative & ESBL-positive & 0 \\
\hline 19 & Negative & Negative & 0 \\
\hline 20 & Negative & Negative & 0 \\
\hline
\end{tabular}

dian milk production per lactation of $8,546 \mathrm{~kg} / \mathrm{cow}$ (interquartile range $=8,189-9,121$; comparable to the Dutch average of all dairy herds). The study herds were randomly located across the country and appeared a representative sample of all Dutch dairy herds (the percentage of study herds was comparable to the percentage of all dairy herds in each of the 12 provinces). From the slurry samples of 41 of the 100 dairy herds, ESBL/ AmpC-producing E. coli were cultured $(41 \% ; 95 \%$ CI $=31-51 \%)$. Thirty-nine of these E. coli isolates were ESBL-producing and 2 were AmpC-producing based on the combination disk diffusion test.

From 5 out of 10 initially positive herds, no ESBL/ AmpC-producing E. coli could be cultured from any of the samples collected during the second visit. From the other 5 initially positive herds, ESBL/AmpCproducing E. coli was cultured from the slurry or from individual cow samples. Whereas only ESBL-producing $E$. coli were cultured from samples collected during the first sampling on 2 of these farms, AmpC-producing E. coli were isolated from both slurry and individual cow samples collected during the second visit. For 4 of 10 initially unsuspected herds, ESBL-producing E. coli were cultured from the slurry to individual cow samples at the second sampling (Table 3).

The within-herd prevalence of herds with ESBL/ AmpC-producing E. coli cultured from slurry samples collected during the second visit $(\mathrm{n}=6)$ ranged from 0 to $100 \%$ with a median of $10 \%$. The within-herd prevalence of herds with no ESBL/AmpC-producing E. coli being cultured from the slurry collected at the second visit $(\mathrm{n}=14)$ ranged from 0 to $13 \%$ with a median of $0 \%$ (Table 3 ).

\section{ESBL/AmpC Genes}

Of the 41 positive slurry samples, $38 \mathrm{E}$. coli isolates that were phenotypically confirmed as ESBL- or AmpC-producing were available for further identification of the $\beta$-lactamase genes. One isolate could not be recultured after storage. In all of the $37 \mathrm{E}$. coli isolates ESBL/AmpC genes were identified (Figure 1). Seven different ESBL genes were found ( $b l a_{\mathrm{CTX}-\mathrm{M}-1,-2,-14,-15,-32,-55}$ and $\left.b l a_{\text {TEM-52 }}\right)$ and only one type of plasmid-coded AmpC gene $\left(b l a_{\mathrm{CMY}-2}\right)$. In 1 of the $5 \mathrm{E}$. coli isolates with an AmpC phenotype, the resistance to cefotaxime was caused by a (inducible) chromosomal $A m p \mathrm{C}$ gene (AmpC type 3$)$. This isolate also carried a non-ESBL TEM gene $\left(b l a_{\text {TEM-1b }}\right)$. One of the $E$. coli isolates with a $b l a_{\text {CTX-M-2 }}$ gene also had a non-ESBL TEM gene: $b l a_{\text {TEM-1a. }}$. The ESBL gene $b l a_{\text {CTX-M-1 }}$ was the most frequently identified gene in this study $(57 \%, 95 \% \mathrm{CI}=$ 39-73), followed by bla $a_{\mathrm{CTX}-\mathrm{M}-15}(13 \%, 95 \% \mathrm{CI}=4-28)$ and $b l a_{\text {CTX-M-2 }}$ and $b l a_{\text {CTX-M-14 }}$ (both $8 \%, 95 \%$ CI $=$ $2-22)$. 


\section{: blaCTX-M-1 II blaCTX-M-15 blaCTX-M-2 $\cong$ blaCTX-M-14 \\ blaCTX-M-32 blaCTX-M-55 — blaTEM-52 blaCMY-2}

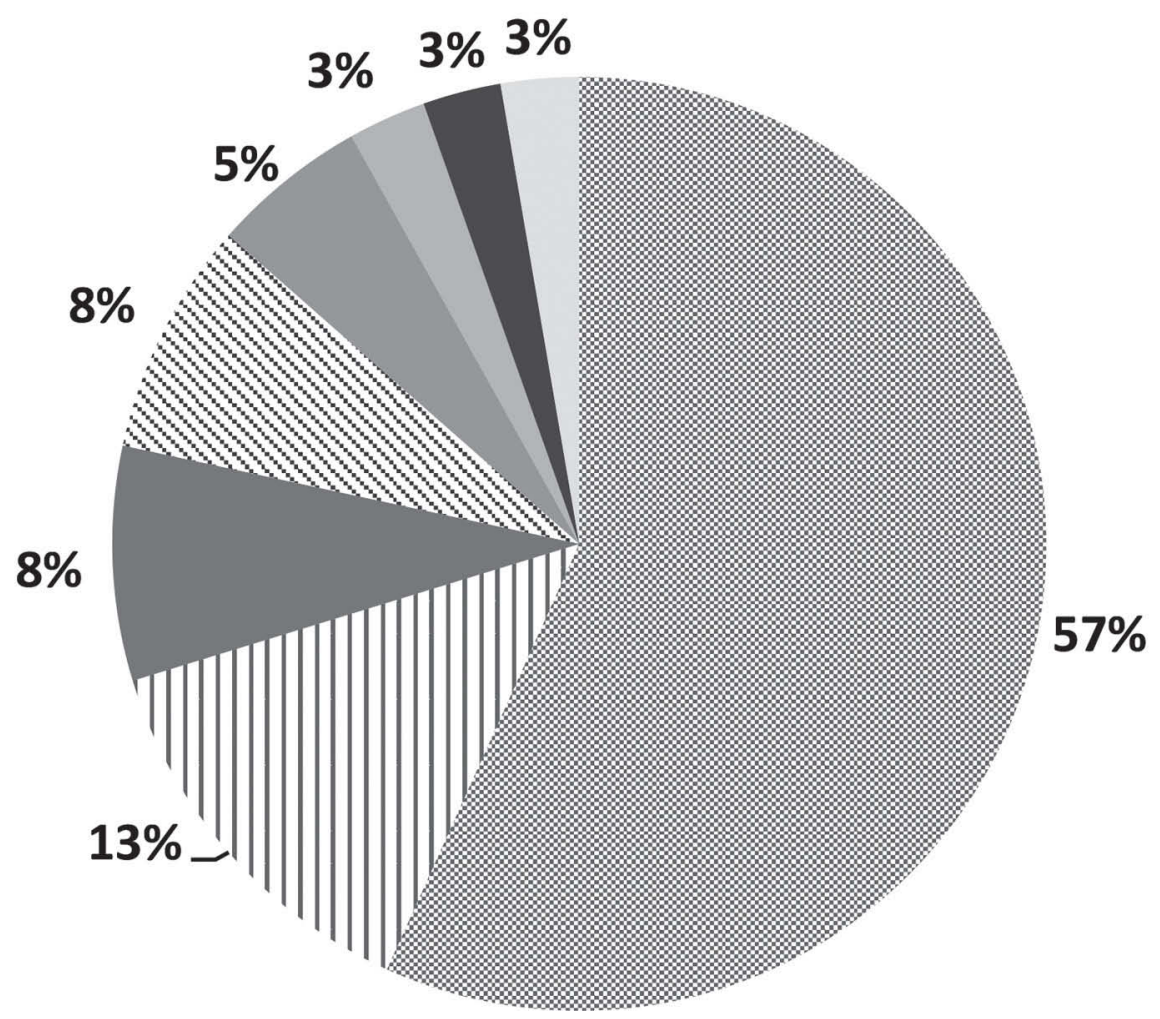

Figure 1. Extended-spectrum $\beta$-lactamase (ESBL) and AmpC genes identified in Escherichia coli isolates from slurry samples from 37 Dutch dairy herds.

\section{Use of Antimicrobials and the Association with ESBL/AmpC Herd Status}

Of the 100 study herds, 4 herds were excluded because of withdrawal of consent by the farmers $(n=2)$ or incompleteness of the data on antimicrobial purchases $(\mathrm{n}=2)$. Eventually, records on 1,139 purchases of antimicrobials in 96 dairy herds were used for analysis. The median total $\mathrm{DDDA}_{\mathrm{F}}$ was 2.55 (mean 2.64) and varied between 0.02 and 6.79 in 2011 . The median total $\mathrm{DDDA}_{\mathrm{F}}$ of 2.71 in ESBL/AmpC-positive dairy herds was not significantly different from the total $\mathrm{DDDA}_{\mathrm{F}}$ in ESBL/AmpC unsuspected herds (median 2.52; Figure 2). The total use of oral antimicrobials, dry cow therapy, and intramammary infusions (in $\mathrm{DDDA}_{\mathrm{F} \text {,oral }}$, $\mathrm{DDDA}_{\mathrm{F}, \mathrm{dry}}$, and $\mathrm{DDDA}_{\mathrm{F} \text {,mast }}$ ) was not significantly different between ESBL/AmpC-positive and unsuspected dairy herds.

The median $\mathrm{DDDA}_{\mathrm{F}}$ (of the total usage) of thirdand fourth-generation cephalosporins in dairy herds, was 0.32 (mean 0.48) in ESBL/AmpC-positive and 0.18 (mean 0.26) in ESBL/AmpC unsuspected herds. In these herds, $1 \mathrm{U}$ increase in $\mathrm{DDDA}_{\mathrm{F}}$ of third- and fourth-generation cephalosporins resulted in a 4.5 times higher odds (95\% CI $=1.4-15.0)$ of being ESBL/AmpCpositive $(P$-value Wald $=0.01)$. The same association was found with the use of intramammary infusions containing third- and fourth-generation cephalosporins. No significant association was found between ESBL/ AmpC status and the use of other groups of antimicrobials, such as (amino)penicillins or others.

\section{Risk Factors for the Presence of ESBL/AmpC- Producing E. coli}

For the risk factor analysis, 96 of the 100 study herds completed the questionnaire and were included in the analyses. The results of the univariable analyses showed that 23 variables were potentially associated ( $P$-value $<0.25$; Wald test) with the probability of a herd being 


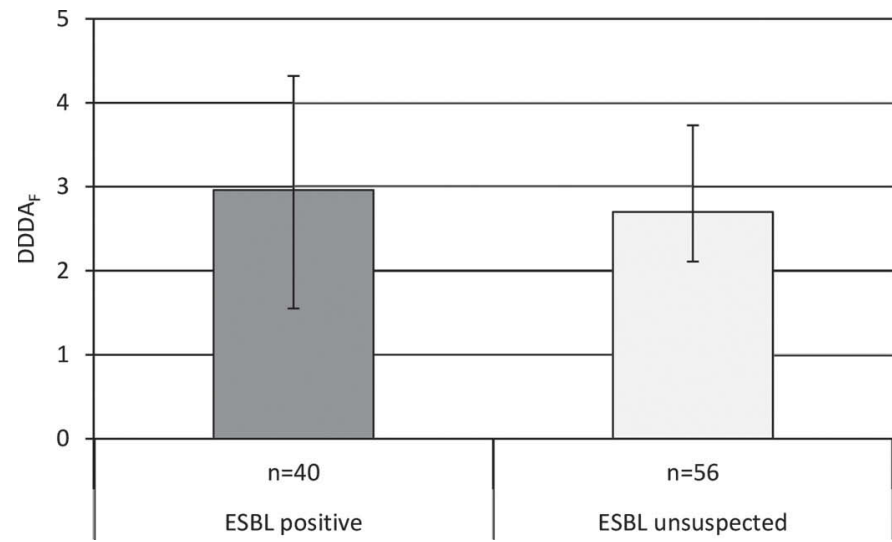

Figure 2. The median and interquartile range of the total animaldefined daily dose per year at farm level $\left(\mathrm{DDDA}_{\mathrm{F}}\right)$ in 2011 for 96 Dutch dairy herds that tested either positive or were unsuspected for extended-spectrum $\beta$-lactamase (ESBL)/AmpC-producing Escherichia coli in slurry.

ESBL/AmpC-positive. The results of the univariable analysis are presented in Appendix Table A1.

The final multivariable model contained 4 parameters and explained $19 \%$ of the variation in ESBL/AmpC status (pseudo- $\mathrm{R}^{2}=0.19$; Table 4 ). When the use of thirdand fouth-generation cephalosporins was added to this model, the explained variation increased to $25 \%$. In this final model, data of 86 herds were included. Records of the 10 excluded herds were incomplete for one or more of the parameters in the final model. Farmers of dairy herds that indicated to treat all cases of clinical mastitis with antimicrobials had a 3.9 times higher odds to have an ESBL/AmpC-positive status than farmers that did not treat all cases (Table 4). Increasing percentages of calves treated with antimicrobials, as indicated by the farmers, resulted in an increased odds of being ESBL/ AmpC-positive. For example, a farmer that estimated that $25 \%$ of the calves were treated with antimicrobials had a 2.09 times higher odds $\left(1.03^{25}\right)$ of having an ESBL/AmpC-positive dairy herd than a farmer that never treated the calves with antimicrobials. Using teat sealants in all cows at dry off had a protective effect on the ESBL/AmpC status. Farmers that used teat sealants in a selected group of cows or farmers that did not use teat sealants at all had, respectively, 7.0 and 4.9 times higher odds of having an ESBL/AmpC-positive herd. Finally, farms where the floors were cleaned with a floor scraper had a 4.6 times higher odds to have an ESBL/AmpC-positive status than farms where the floors were cleaned with other methods.

For 57 of the 100 study herds, the prescribed amount of teat sealants was available. Running the logistic regression models with the prescribed amount of teat sealants instead of the results of the questionnaire, the association with ESBL/AmpC herd status that was found using the questionnaire results, could not be confirmed.

\section{DISCUSSION}

This study estimated the between-herd prevalence of ESBL/AmpC-producing E. coli in dairy herds at $41 \%(95 \%$ CI $=31-51 \%)$. This is the first time the

Table 4. Results of the multivariable logistic regression analysis modeling the probability of testing positive for extended-spectrum $\beta$-lactamase (ESBL)/AmpC-producing Escherichia coli in 86 Dutch dairy farms in 2011, based on the results from a questionnaire

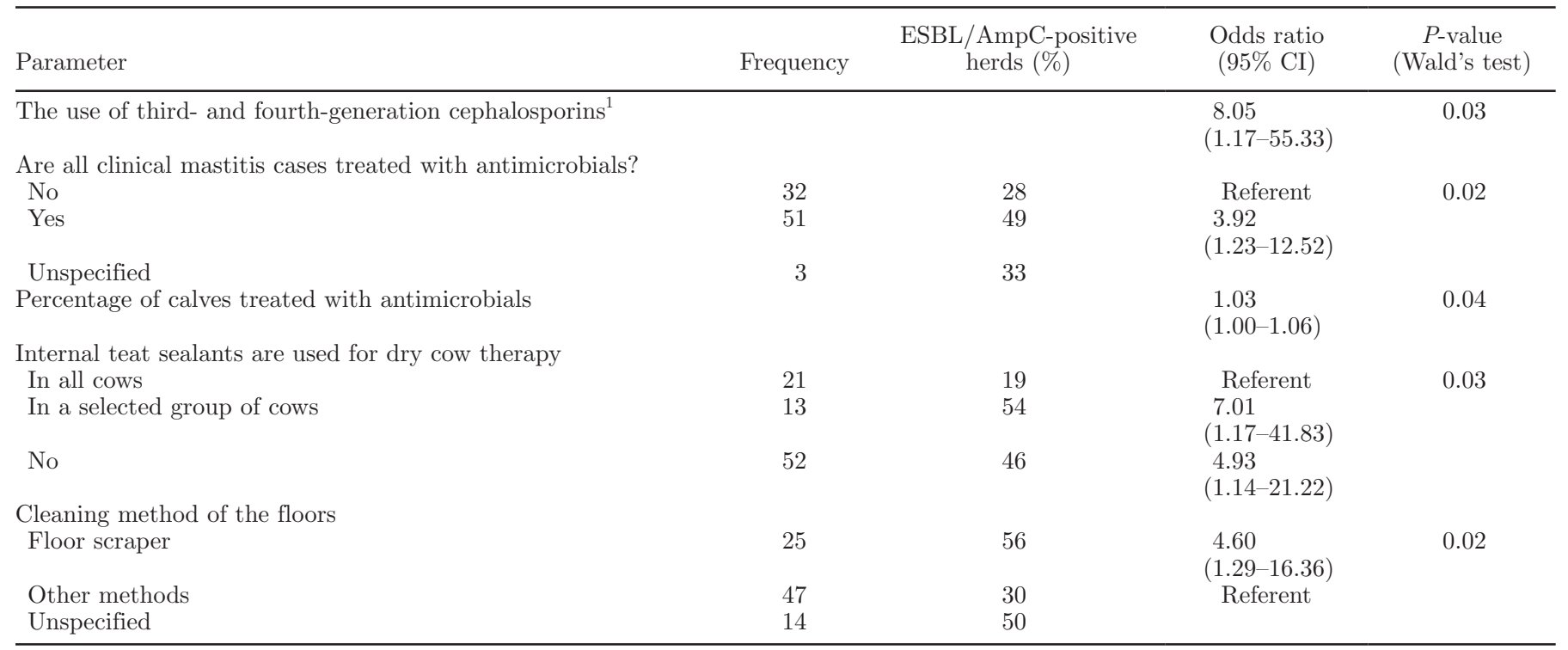

\footnotetext{
${ }^{1}$ This group of antimicrobials included the use of cefoperazone, cefquinome, and ceftiofur.
} 
herd prevalence of ESBL/AmpC -producing E. coli was established among dairy herds in the Netherlands. In recent studies conducted in other countries prevalences among dairy herds varied from 5 to $87 \%$ (Snow et al., 2012; Ohnishi et al., 2013; Schmid et al., 2013). However, these studies vary in, among others, the use of different selective media and the selection of farms, which results in different prevalences of ESBL/AmpCproducing E. coli.

One of the aims of our study was to evaluate risk factors for ESBL/AmpC on a herd level. This information is important to study management practices related to the occurrence of ESBL/AmpC. Because bacteriological culturing and typing of ESBL/AmpC is relatively expensive, most studies are based on a small number of herds (Tragesser et al., 2006; Mollenkopf et al., 2012). Sample size calculations showed that at least 100 herds have to be included in a study to enable detection of risk factors with an odds ratio of 3.5 or higher $(20 \%$ estimated exposure rate in the control group). Thus, given our budgetary limitations, we decided to determine the ESBL/AmpC herd status based on herd-level slurry samples. Based on earlier work from Lombard et al. (2012) and Schmid et al. (2013), pooled slurry samples were expected to be sensitive enough to determine the ESBL/AmpC herd status. Specific attention was given to take the slurry samples from different sites in the herd to contain $E$. coli isolates originating from multiple cows; the presence of ESBL/AmpC-producing $E$. coli on a farm can be underestimated using this sampling method. This was supported by results of the second visit: on 3 out of 14 farms where no ESBL/ AmpC-producing E. coli were cultured from the slurry, these bacteria were cultured from 1 (2 farms) or 3 cows (1 farm). A negative test result from the slurry sample does not mean that all animals in the herd are free of ESBL/AmpC-producing E. coli; these bacteria can still be present, although apparently in low within-herd prevalences, among the cows. Therefore, we decided to define herds that tested negative based on the slurry sample as unsuspected and not as negative. In 2 herds that tested ESBL/AmpC-positive based on the slurry sample, all of the 24 individual cows tested negative, indicating that individual sampling of 24 cows is also not $100 \%$ sensitive. Thus, with either sampling method some misclassification bias may occur. Misclassification bias mainly leads to underestimation of the associations of the explanatory variable with the outcome. Therefore, we believe that the risk factors that were detected with this study represent true risk factors for the ESBL/AmpC herd status.

The most frequently found ESBL genotypes in our study originated from CTX-M group 1 ( $b l a_{\text {CTX-M-1 }}$, $b l a_{\mathrm{CTX}-\mathrm{M}-15}, b l a_{\mathrm{CTX}-\mathrm{M}-32}$ and $\left.b l a_{\mathrm{CTX}-\mathrm{M}-55}\right)$. Genes from this group were also identified most frequently in previous studies in which ESBL-producing E.coli isolates from cattle were sequenced in Great Britain (Snow et al., 2012; Wu et al., 2013), the Netherlands (Wu et al., 2013), Germany (Wu et al., 2013), and Switzerland (Geser et al., 2012). Other frequently found ESBL genotypes belonged to CTX-M group $2\left(b l a_{\mathrm{CTX}-\mathrm{M}-2}\right)$ or CTX-M group 9 ( $\left.b l a_{\text {CTX-М-14 }}, b l a_{\text {CTX-М-27 }}\right)$, which were also often found in isolates from cattle in previous European studies (Geser et al., 2012; Wu et al., 2013; Valentin et al., 2014). The genotypes $b l a_{\mathrm{TEM}-52}$ and $b l a_{\mathrm{CMY}-2}$, which were identified in only one of the sequenced isolates in our study, were also sporadically found in isolates from cattle in previous European research (Hordijk et al., 2013b; Wu et al., 2013; Carmo et al., 2014). These latter genotypes, however, appeared to occur more often in chicken and humans than in cattle (Wu et al., 2013). The genotypes of ESBL/AmpC and their distribution found in our study appeared comparable to genotypes that were found in previous research conducted in cattle.

In dairy herds, the use of third- and fourth-generation cephalosporins was positively associated with the ESBL/AmpC status. Snow et al. (2012) also found that the use of third- and fourth-generation cephalosporins resulted in a higher probability to be ESBL/AmpCpositive in dairy farms. In addition, Tragesser et al. (2006) also found an association between the use of ceftiofur (third-generation cephalosporins) and the presence of $E$. coli with reduced susceptibility to ceftriaxone among cattle on dairy farms. In the Netherlands, the government has restricted the use of third- and fourth-generation cephalosporins since 2013 (Speksnijder et al., 2015; Kuipers et al., 2016). Currently, before a veterinarian decides to treat a cow with these antimicrobials, he or she has to prove with bacteriological culturing and sensitivity testing that there is no other alternative. This has resulted in a steep decrease in the use of this type of antimicrobial toward negligible levels in 2014 (SDa, 2015). The attitude of farmers to treat all clinical mastitis cases with antimicrobials was also associated with a positive ESBL/AmpC herd status.

No association was found between the total AMU and the ESBL/AmpC herd status. Unfortunately, no information was available on the actually treated animals on the farms. Oral antimicrobials can only be administered to calves and not to other cattle, and can therefore be allocated with certainty to the calves. Antimicrobials for parenteral use can, however, be administered to calves as well as to older cattle, which can result in an underestimation of the amount of antimicrobials administered to calves. The association found, based on the questionnaire data on estimated AMU in calves, could therefore not be confirmed with an analyses of 
the association between prescribed AMU in calves and the ESBL/AmpC herd status.

The questionnaire conducted among the farmers also revealed an association between the use of teat sealants and the ESBL/AmpC herd status: applying teat sealant in all cows at dry off seemed protective. This association, however, could not be confirmed with the prescribed amount of teat sealants. An explanation for these different results could be the difference in number of included herds. Only 57 herd invoices of teat sealants were available, compared with 86 herds included in the model with the questionnaire data. Because of the differences in results, the association found based on the questionnaire data on the use of teat sealants were not fully convincing and should be studied in more detail.

The use of a floor scraper was associated with ESBL/ AmpC in slurry samples, which might be partly explained by the sampling methodology. In our study, 2 sampling methods were applied: sampling the floor scraper, and sampling 5 different places on the slatted floor in herds without a floor scraper. A scraper is likely contaminated with manure from multiple if not all cattle in the barn. This sample is therefore assumed to be relatively sensitive to detect $\mathrm{ESBL} / \mathrm{AmpC}$ on a herd level based on a single sample. To prevent selection bias, we did not want to exclude herds without a floor scraper from our study and it was decided to develop an alternative sampling method for these herds. Our results showed that the scraper samples were more often ESBL/AmpC-positive compared with the pooled samples from manure of the slatted floor. Nevertheless, herds with a high within-herd prevalence seemed to be detected with either method, and false negative herdlevel results only occurred in herds with a low withinherd prevalence. The final multivariable model contained explained $19 \%$ of the variation in ESBL/AmpC herd status, which means that $82 \%$ of the variation in ESBL/AmpC herd status is attributable to factors that were not evaluated in our study. Although the variation in ESBL/AmpC herd status that was explained by this model was limited, the risk factors were in line with those that were described earlier (Tragesser et al., 2006; Snow et al., 2012).

Based on earlier work of Ojer-Usoz et al. (2013), it is known that beef can be contaminated with ESBL. Nevertheless, to which extent the ESBL/AmpC herd status found in our study was associated with possible contamination of meat was not evaluated.

Little is known about the dynamics of ESBL-producing E. coli at animal and herd level in dairy cattle, including the levels and periods of shedding. Hordijk et al. (2013a) studied the prevalence of ESBL-producing E. coli among veal calves on 3 farms during the first 10 wk of a production cycle. The prevalence declined from
18 to $26 \%$ at the moment of arrival, to 0 to $1.4 \%$ after 10 wk. These results showed that most of the previously positive calves were no longer colonized by ESBLproducing E. coli after 10 wk (Hordijk et al. 2013a). In humans, different periods of excretion were found as well, ranging from 2 to almost 7 mo (Apisarnthanarak et al., 2008; Li et al., 2012; Birgand et al., 2013). The results of our study could also indicate that the presence of ESBL/AmpC-producing E. coli in slurry samples changes over time. In 6 out of 10 initially positive herds, no ESBL- or AmpC-producing E. coli could be cultured from slurry samples collected during the second visit, whereas the other 4 farms remained positive in the slurry. In 2 out of 10 initially unsuspected herds, ESBL/AmpC-producing E. coli were cultured from the slurry during the second visit. This change in status could, however, also be due to the relatively low sensitivity of our sampling method. Therefore, it is recommended to conduct further research to gain more insight in the period ESBL/AmpC-producing $E$. coli are present in dairy herds and how these bacteria spread among dairy cows, young stock and calves.

\section{CONCLUSIONS}

This is the first study that estimated the herd-level prevalence of ESBL- and AmpC-producing E. coli among Dutch dairy farms. The ESBL/AmpC-producing E. coli isolates carried the same ESBL/AmpC genes as have been found in cattle isolates in previous research conducted in the Netherlands and in other European countries. No association was found between the total AMU and the ESBL/AmpC herd status. The use of third- and fourth-generation cephalosporins, however, was associated with an increased odds of having a positive ESBL/AmpC herd status and seems important in reducing ESBL/AmpC. Four other management factors were also found to be associated with the ESBL/AmpC status of dairy herds. Further research should focus on the dynamics of ESBL/AmpC-producing E. coli within dairy herds.

\section{ACKNOWLEDGMENTS}

This study was financed by the Dutch Commodity Board for Dairy (PZ, The Hague, the Netherlands). We thank all farmers for their participation and their commitment to this study. Furthermore, we thank Leo Dekkers (GD Animal Health, Deventer, the Netherlands) for organizing all the logistics during the project.

\section{REFERENCES}

Akaike, H. 1974. A new look at the statistical model identification. IEEE Trans. Automat. Contr. 19:716-723. 
Apisarnthanarak, A., T. C. Bailey, and V. J. Fraser. 2008. Duration of stool colonization in patients infected with extended-spectrum $\beta$-lactamase-Producing Escherichia coli and Klebsiella pneumonia. Clin. Infect. Dis. 46:1322-1323.

Birgand, G., L. Armand-Lefevre, I. Lolom, E. Ruppe, A. Andremont, and J. C. Lucet. 2013. Duration of colonization by extended-spectrum $\beta$-lactamase-producing Enterobacteriaceae after hospital discharge. Am. J. Infect. Control 41:443-447.

Carattoli, A., A. Garcia-Fernandez, P. Varesi, D. Fortini, S. Gerardi, A. Penni, C. Mancini, and A. Giordano. 2008. Molecular epidemiology of Escherichia coli producing extended-spectrum betalactamases isolated in Rome, Italy. J. Clin. Microbiol. 46:103-108.

Carmo, L. P., L. R. Nielsen, P. M. da Costa, and L. Alban. 2014. Exposure assessment of extended-spectrum beta-lactamases/AmpC beta-lactamases-producing Escherichia coli in meat in Denmark. Infect. Ecol. Epidemiol. 4:22924. http://dx.doi.org/10.3402/iee. $\mathrm{v} 4.22924$.

Clinical and Laboratory Standards Institute (CLSI). 2011. Performance Standards for Antimicrobial Susceptibility Testing: Twenty-first Informational Supplement M100-21. CLSI, Wayne, PA.

Dierikx, C., A. van Essen-Zandbergen, K. Veldman, H. E. Smith, and D. J. Mevius. 2010. Increased detection of extended spectrum beta-lactamase producing Salmonella enterica and Escherichia coli isolates from poultry. Vet. Microbiol. 145:273-278.

Dierikx, C. M., J. A. van der Goot, H. E. Smith, A. Kant, and D. J. Mevius. 2013. Presence of ESBL/AmpC-producing Escherichia coli in the broiler production pyramid: a descriptive study. PLoS ONE 8:e79005. http://dx.doi.org/10.1371/journal.pone.0079005.

Dierikx, C. M., E. van Duijkeren, A. H. Schoormans, A. van EssenZandbergen, K. Veldman, A. Kant, X. W. Huijsdens, K. van der Zwaluw, J. A. Wagenaar, and D. J. Mevius. 2012. Occurrence and characteristics of extended-spectrum- $\beta$-lactamase- and AmpCproducing clinical isolates derived from companion animals and horses. J. Antimicrob. Chemother. 67:1368-1374.

EFSA. 2011. EFSA Panel on Biological Hazards: Scientific opinion on the public health risks of bacterial strains producing extended-spectrum $\beta$-lactamases and/or AmpC $\beta$-lactamases in food and food-producing animals. EFSA J. 9:2322. http://dx.doi. org/10.2903/j.efsa.2011.2322.

Ewers, C., A. Bethe, T. Semmler, S. Guenther, and L. H. Wieler. 2012. Extended-spectrum b-lactamase-producing and AmpC-producing Escherichia coli from livestock and companion animals, and their putative impact on public health: A global perspective. Clin. Microbiol. Infect. 18:646-655. http://dx.doi.org/10.1111/j.14690691.2012.03850.x.

Geser, N., R. Stephan, and H. Hächler. 2012. Occurrence and characteristics of extended-spectrum b-lactamase (ESBL) producing Enterobacteriaceae in food producing animals, minced meat and raw milk. BMC Vet. Res. 8:21. http://dx.doi.org/10.1186/1746$6148-8-21$

Hawkey, P. M., and A. M. Jones. 2009. The changing epidemiology of resistance. . J. Antimicrob. Chemother. 64:i3-i10. http://dx.doi. org/10.1093/jac/dkp256.

Hordijk, J., J. A. Wagenaar, A. Kant, A. van Essen-Zandbergen, C. M. Dierikx, K. Veldman, B. Wit, and D. J. Mevius. 2013a. Within-farm dynamics of ESBL/AmpC producing Escherichia coli in veal calves: A longitudinal approach. . J. Antimicrob. Chemother. 68:2468-2476.

Hordijk, J., J. A. Wagenaar, A. van de Giessen, C. M. Dierikx, A. van Essen-Zandbergen, K. Veldman, A. Kant, and D. J. Mevius. 2013b. Increasing prevalence and diversity of ESBL/AmpC-type $\beta$-lactamase genes in Escherichia coli isolated from veal calves from 1997 to 2010. J. Antimicrob. Chemother. 68:1970-1973.

Korzeniewska, E., A. Korzeniewska, and M. Harnisz. 2013. Antibiotic resistant Escherichia coli in hospital and municipal sewage and their emission to the environment. Ecotoxicol. Environ. Saf. 91:96-102.

Kuipers, A., W. J. Koops, and H. Wemmenhove. 2016. Antibiotic use in dairy herds in the Netherlands from 2005 to 2012. J. Dairy Sci. 99:1632-1648.
Lahey. 2012. $\beta$-Lactamase Classification and Amino Acid Sequences for TEM, SHV and OXA Extended-Spectrum and Inhibitor Resistant Enzymes. Accessed March 2012. http://www.lahey.org/ studies.

Laube, H., A. Friese, C. von Salviati, B. Guerra, and U. Rösler. 2014. Transmission of ESBL/AmpC-producing Escherichia coli from broiler chicken farms to surrounding areas. Vet. Microbiol. 172:519-527

Li, B., Y. Zhong, X.-C. Fu, Y.-H. Qiu, S.-Y. Wang, A.-J. Yang, and X.-H. Huang. 2012. Duration of stool colonization in healthy medical students with extended-spectrum-B-lactamase-producing Escherichia coli. Antimicrob. Agents Chemother. 56:4558-4559.

Lombard, J. E., A. L. Beam, E. M. Nifong, C. P. Fossler, C. A. Kopral, D. A. Dargatz, B. A. Wagner, M. M. Erdman, and P. J. FedorkaCray. 2012. Comparison of individual, pooled, and composite fecal sampling methods for detection of Salmonella on U.S. dairy operations. J. Food Prot. 75:1562-1571.

Mollenkopf, D. F., M. F. Weenman, J. B. Daniels, M. J. Abley, J. L. Mathews, and W. A. Gebreyes. 2012. Variable within- and between-herd diversity of CTX-M cephalosporinase-bearing Escherichia coli isolates from dairy cattle. Appl. Environ. Microbiol. $78: 4552-4560$.

Ohnishi, M., A. T. Okatani, H. Esaki, K. Harada, T. Sawada, M. Murakami, K. Marumo, Y. Kato, R. Sato, K. Shimura, N. Hatanaka, and T. Takahashi. 2013. Herd prevalence of Enterobacteriaceae producing CTX-M-type and CMY-2 $\beta$-lactamases among Japanese dairy farms. J. Appl. Microbiol. 115:282-289.

Ojer-Usoz, E., D. González, A. I. Vitas, J. Leiva, I. Garcìa-Jalón, A. Febles-Casquero, and M. L. Escolano. 2013. Prevalence of extended-spectrum $\beta$-lactamase-producing Enterobacteriaceae in meat products sold in Navarra, Spain. Meat Sci. 93:316-321.

Pitout, J. D. D., and K. B. Laupland. 2008. Extend-spectrum beta-lactamase-producing Enterobacteriaceae: An emerging public-health concern. Lancet Infect. Dis. 8:159-166.

Schmid, A., S. Hörmansdorfer, U. Messelhäusser, A. Käsbohrer, C. Sauter-Louis, and R. Mansfeld. 2013. Prevalence of extended-spectrum $\beta$-lactamase-producing Escherichia coli on Bavarian dairy and beef cattle farms. Appl. Environ. Microbiol. 79:3027-3032.

Scientific Advisory Group on Antimicrobials of the Committee for Medicinal Products. 2009. Reflection paper on the use of third and fourth generation cephalosporins in food producing animals in the European Union: Development of resistance and impact on human and animal health. J. Vet. Pharmacol. Ther. 32:515-533.

SDa. 2015. Usage of antibiotics in agricultural livestock in the Netherlands in 2014. Trends and benchmarking of livestock farms and veterinarians. Accessed Sep. 7, 2016. http://www. autoriteitdiergeneesmiddelen.nl/Userfiles/pdf/SDa-rapporten/defrapport-sda-het-gebruik-van-antibiotica-bij-landbouwhuisdierenin-2014-incl-erratum.pdf.

Snow, L. C., R. G. Warner, T. Cheney, H. Wearing, M. Stokes, K. Harris, C. J. Teale, and N. G. Coldham. 2012. Risk factors associated with extended spectrum beta-lactamase Escherichia coli (CTX-M) on dairy farms in North West England and North Wales. Prev. Vet. Med. 106:225-234.

Speksnijder, D. C., D. J. Mevius, C. J. Bruschke, and J. A. Wagenaar. 2015. Reduction of veterinary antimicrobial use in the Netherlands. The Dutch success model. Zoonoses Public Health 62:79-87.

StataCorp. 2014. Stata Software version 13. Stata Corporation, College Station, TX.

Thrusfield, M., C. Ortega, I. de Blas, J. P. Noordhuizen, and K. Frankena. 2001. WIN EPISCOPE 2.0: improved epidemiological software for veterinary medicine. Vet. Rec. 148:567-572.

Tragesser, L. A., T. E. Wittum, J. A. Funk, P. L. Winokur, and P. J. Rajala-Schultz. 2006. Association between ceftiofur use and isolation of Escherichia coli with reduced susceptibility to ceftriaxone from fecal samples of dairy cows. Am. J. Vet. Res. 67:1696-1700.

Valentin, L., H. Sharp, K. Hille, U. Seibt, J. Fischer, Y. Pfeifer, G. B. Michael, S. Nickel, J. Schmiedel, L. Falgenhauer, A. Friese, R. Bauerfeind, U. Roesler, C. Imirzalioglu, T. Chakraborty, R. Helmuth, G. Valenza, G. Werner, S. Schwarz, B. Guerra, B. Appel, 
L. Kreienbrock, and A. Käsbohrer. 2014. Subgrouping of ESBLproducing Escherichia coli from animal and human sources: an approach to quantify the distribution of ESBL types between different reservoirs. Int. J. Med. Microbiol. 304:805-816. http://dx.doi. org/10.1016/j.ijmm.2014.07.015.

von Salviati, C., H. Laube, B. Guerra, U. Roesler, and A. Friese. 2015. Emission of ESBL/AmpC-producing Escherichia coli from pig fattening farms to surrounding areas. Vet. Microbiol. 175:77-84.

WHO. 2012. Critically important antimicrobials for human medicine, third revision. Accessed May 6, 2016. http://www.who.int/ foodsafety/publications/antimicrobials-third/en/.
Wu, G., M. J. Day, M. T. Mafura, J. Nunez-Garcia, J. J. Fenner, M. Sharma, A. van Essen-Zandbergen, I. Rodríguez, C. Dierikx, K. Kadlec, A. K. Schink, M. Chattaway, J. Wain, R. Helmuth, B. Guerra, S. Schwarz, J. Threlfall, M. J. Woodward, N. Woodford, N. Coldham, and D. Mevius. 2013. Comparative analysis of ESBLpositive Escherichia coli isolates from animals and humans from the UK, The Netherlands and Germany. PLoS ONE 8:e75392 http://dx.doi.org/10.1371/journal.pone.0075392.

\section{APPENDIX}

Table A1. Descriptive results (no. with \% in parentheses unless otherwise noted) of the univariable logistic regression analyses between extended-spectrum $\beta$-lactamase (ESBL)/AmpC status and management factors in 96 dairy farms

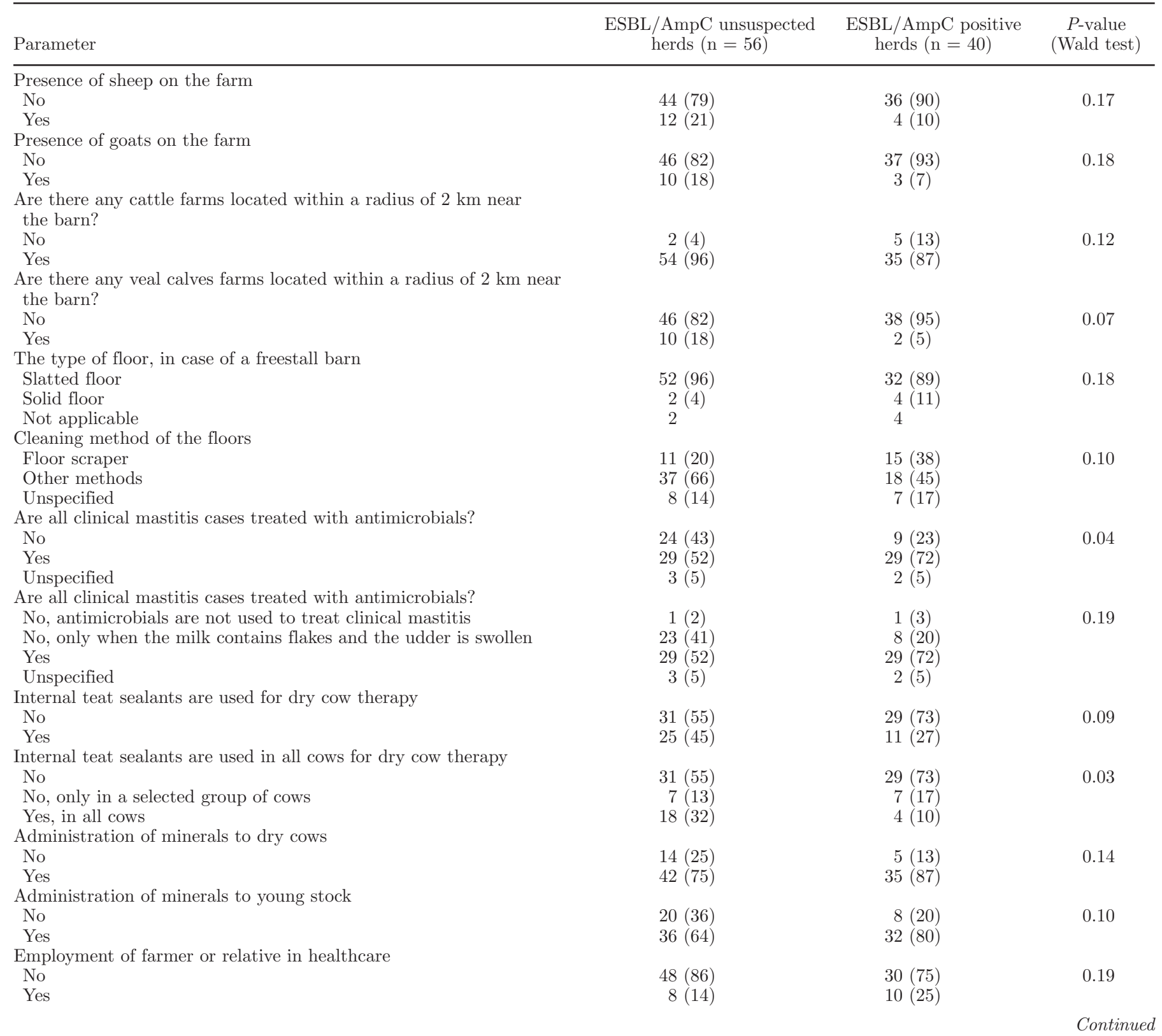


Table A1 (Continued). Descriptive results (no. with \% in parentheses unless otherwise noted) of the univariable logistic regression analyses between extended-spectrum $\beta$-lactamase (ESBL)/AmpC status and management factors in 96 dairy farms

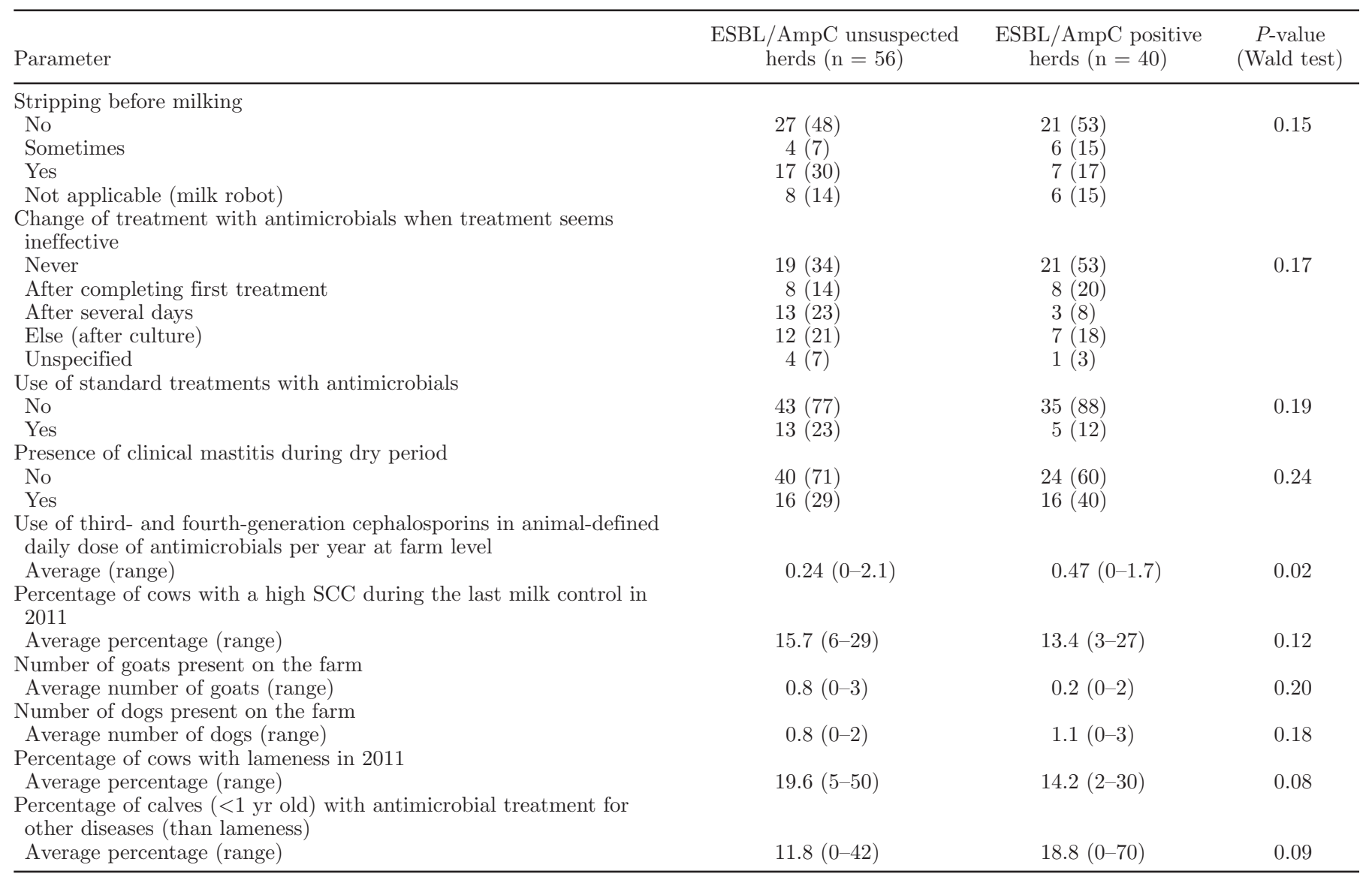

